\title{
Percepción del estudiantado chileno de enseñanza secundaria hacia distintos tipos de Feedback Correctivo Oral (FCO) en errores de pronunciación: Un análisis cualitativo
}

Orellana Marihuén, Paula Francisca; Ortiz Navarrete, Mabel Andrea

Percepción del estudiantado chileno de enseñanza secundaria hacia distintos tipos de Feedback Correctivo Oral (FCO) en errores de pronunciación: Un análisis cualitativo

Revista Educación, vol. 45, núm. 2, 2021

Universidad de Costa Rica, Costa Rica

Disponible en: https://www.redalyc.org/articulo.oa?id=44066178041

DOI: https://doi.org/10.15517/revedu.v45i1.43636

\section{(c) $\odot \Theta \Theta$}

Esta obra está bajo una Licencia Creative Commons Atribución-NoComercial-SinDerivar 3.0 Internacional. 


\title{
Percepción del estudiantado chileno de enseñanza secundaria hacia distintos tipos de Feedback Correctivo Oral (FCO) en errores de pronunciación: Un análisis cualitativo
}

\author{
Perceptions of Eleventh Grade EFL Students at a Public High School in Chile Regarding Different Types of Oral \\ Corrective feedback (OCF) Pronunciation Errors: A Qualitative Analysis.
}

Paula Francisca Orellana Maribuén

Universidad Católica de la Santísima Concepción, Chile

porellana@magisteredu.ucsc.cl

(iD https://orcid.org/0000-0002-2541-9655

\author{
Mabel Andrea Ortiz Navarrete \\ Universidad Católica de la Santísima Concepción, Chile \\ mortiz@ucsc.cl \\ (iD https://orcid.org/0000-0001-7481-1293
}

DOI: https://doi.org/10.15517/revedu.v45i1.43636 Redalyc: https://www.redalyc.org/articulo.oa? $\mathrm{id}=44066178041$

\section{Resumen:}

El presente artículo tiene como objetivo analizar la percepción de estudiantes de tercer año medio, de un establecimiento educacional de la provincia de Concepción hacia distintos tipos de retroalimentación ofeedback correctivo oral en errores de pronunciación. La metodología utilizada se basa en el enfoque de investigación cualitativa de paradigma interpretativo bajo un diseño de estudio de caso. La muestra estuvo constituida por 18 estudiantes de tercer año medio, a quienes se les aplicó una entrevista semiestructurada e individual. Mediante el análisis del contenido se obtuvo que las personas consideran al feedback como un aspecto favorable para su aprendizaje, particularmente para mejorar su pronunciación. Ellos y ellas se inclinan por la modalidad implícita de corrección y prefieren la estrategia de feedback de tipo pista metalingüística. Además, consideran que el mejor momento para recibir este feedback es inmediatamente finalizada la tarea oral. Este estudio busca resaltar la importancia del Feedback Correctivo Oral y su beneficio en aspectos de la oralidad, como en la pronunciación del estudiantado que aprende inglés como lengua extranjera.

Palabras ClaVe: Pronunciación, Retroalimentación, Inglés como lengua extranjera, Enseñanza secundaria.

\section{AbStract:}

This study explores the perception of 11 th grade EFL students at a public high school in the Concepcion, Chile school district regarding various types of Oral Corrective Feedback (OCF) for pronunciation mistakes. A qualitative research methodology is used with an interpretative paradigm and a case study design. The study sample included 18 high school students in Concepción who participated in a one-on-one semi-structured interview. According to results attained through content analysis, learners considered feedback as beneficial for learning, especially for improving pronunciation. OCF student preferences included implicit feedback and metalinguistic clue strategies. Students also perceived OCF as most effective immediately after concluding the speaking task. This overall aim of the study is to highlight the importance of OCF and its contributions to helping EFL students develop their speaking skills.

KEYWORDS: Pronunciation, Feedback, English as a Foreign Language, High School Education.

\section{INTRODUCCIÓN}

Las bases curriculares que rigen la educación del inglés como lengua extranjera en Chile plantean, en el año 2019, la importancia de desarrollar en el estudiantado chileno la habilidad de comunicar mensajes orales, 
con una pronunciación adecuada y con la claridad necesaria para poder participar de intercambios hablados (Ministerio de educación chileno [MINEDUC], 2019b).

En este contexto, diversas personas investigadoras y especialistas de la enseñanza del inglés, ya sea como segunda lengua o como lengua extranjera (Brown, 2001; Nunan, 2003; Ur, 2009), han considerado la habilidad hablada, o el speaking, como la habilidad lingüística más difícil de desarrollar en una sala de clases, particularmente el aspecto de la pronunciación. Varios estudios extranjeros han hecho hincapié en la necesidad de investigar cómo la corrección o retroalimentación de la habilidad oral puede favorecer el aprendizaje y producción del inglés (Sheen, 2010a; Sheen, 2010b; Atma y Widiati, 2016); sin embargo, existen muy pocos estudios en Chile en esta área de la evaluación de la lengua (Aránguiz y Quintanilla, 2016; Muñoz y Ferreira, 2017).

En relación con la retroalimentación de la pronunciación, Huang y Jia (2016) mencionan que es escasa la investigación en este ámbito; hacen hincapié en la necesidad de analizar las percepciones del estudiantado en torno a la retroalimentación de la pronunciación y la consideran pedagógicamente importante para la enseñanza de lenguas extranjeras. Diversas investigaciones comprueban que el análisis en torno a la percepción estudiantil es bajo, ya que la mayoría de los estudios están enfocados en conocer la perspectiva docente respecto al proceso de retroalimentación oral (Hattie y Timperley, 2007; Poulos y Mahony, 2008; Canabal y Margalef, 2017). En relación a los estudios nacionales encontrados, no se habla de las preferencias o percepciones estudiantiles, por lo que existe un vacío de conocimiento en este ámbito (Aránguiz y Quintanilla, 2016; Muñoz y Ferreira, 2017).

Es necesario destacar que la percepción del estudiantado le da validez a la retroalimentación en la medida en que se considera su voz en el proceso de enseñanza. De acuerdo con estudios extranjeros, particularmente el de Atma y Widiati (2016), el conocer las preferencias del estudiantado en torno a la habilidad oral y sus componentes, permite que el aprendizaje sea cercano, contextualizado y significativo para este.

En conclusión, este estudio pretende promover la reflexión en torno a las estrategias de corrección que favorecen la pronunciación del estudiantado a partir de sus percepciones.

\section{REVISIÓN DE LA LITERATURA}

\section{La pronunciación en idioma inglés}

Selinker (1972) elaboró la primera teoría refiriéndose a la interlengua; menciona que es aquel idioma que está en proceso de desarrollo. Esto implica ciertos factores que influyen en el logro de la L2, tal como indica Ellis (2017a), quien recalca que la lengua materna puede influir enormemente el aprendizaje de la L2, por lo tanto, ejerce un rol predominante en la interlengua.

Enseñar pronunciación es muy importante porque ella individualiza los idiomas, los hace únicos. Kenworthy (1987) cree que aprendices de una segunda lengua adquieren tonos de pronunciación dependiendo de sus necesidades, propósitos e intereses.

La pronunciación que genera una fácil comprensión del mensaje es un aspecto fundamental en términos de demostración del dominio de un idioma. Según Pourhosein (2012), la pronunciación es un aspecto que, si bien es relevante para estudiantes de la lengua, corresponde a un proceso, por lo tanto, lo que se espera, no es la perfección. Marzá (2014) manifiesta que el proceso de enseñanza-aprendizaje de la pronunciación del inglés como lengua extranjera juega un rol fundamental desde la base que desarrolla competencias comunicativas de la persona hablante. 


\section{Los errores en pronunciación}

El escenario educativo es transformador, es decir, un error es algo temporal que puede y debe ser analizado, consensuado, examinado, evaluado y retroalimentado. Según Aránguiz y Quintanilla (2016), la dificultad en la producción oral dentro de las aulas chilenas no es algo nuevo. "Los estudiantes normalmente manifiestan el no querer participar de actividades habladas porque sienten no tener las herramientas para tener una comunicación fluida, además que no quieren cometer errores" (p.105). Como mencionan las autoras, es importante que cada persona que esté aprendiendo un idioma sea corregida en algún momento del proceso de aprendizaje, puesto que "la corrección del error es una parte natural de la adquisición de una lengua extranjera” (Azar, 2007 citado en Aránguiz y Quintanilla 2016, p. 105).

Para ejemplificar, algunos estudios relacionados con la pronunciación señalan que los errores más comunes que comete el estudiantado, son los errores de tipo acentual, es decir, errores en la acentuación de palabras, lo que interfiere en la comprensión del mensaje (Bohn y Hansen, 2017). Otros estudios señalan que algunas personas estudiantes cometen errores de pronunciación al intentar alcanzar la naturalidad simulando a un hablante nativo, ya que la imitación se hace simulando sonidos, sin un conocimiento técnico de aquellos (Baker y Burri, 2016). De acuerdo a las personas autoras, es más difícil adoptar una similitud a un hablante nativo de la L2 en estudiantes adolescentes, puesto que existen una serie de factores que pueden avergonzar la pronunciación ideal, aunque sí se tengan las habilidades para ello. En palabras comunes, es más aceptado tener ciertos errores naturalizados que hablar con una pronunciación perfecta, ya que es socialmente aceptado en su entorno.

En la actualidad, cometer un error es algo que sucede a diario dentro del aula y corresponde a un proceso a mejorar paulatinamente. Cuando el profesor sugiere la entrega de una corrección que vaya más allá de la mera corrección, se está frente al concepto de retroalimentación ofeedback, en inglés.

\section{Retroalimentación o feedback}

Varias personas autoras han considerado al feedbackcomo un elemento central dentro del proceso de aprendizaje de una lengua (Ellis y Sheen, 2006; Sheen y Ellis, 2011; Aránguiz y Quintanilla, 2016; Shabani y Safari, 2016, Quinn y Nakata, 2017, Ellis, 2017b; Hanh y Tho, 2018). Como afirman Muñoz y Ferreira (2017), "el feedback es definido como la información que se proporciona a los estudiantes" (p. 75). Esto se hace con el fin de que el alumnado ponga atención a los distintos factores que puedan estar afectando la emisión correcta de un mensaje, en el caso de la oralidad. Según Hanh y Tho, (2018), el "feedback puede ser positivo o negativo dependiendo si el profesor quisiera enfrentar o premiar al estudiante o incluso corregir sus errores" (p.40). Por lo tanto, la relación inmediata al concepto de feedback es el feedback correctivo (FC), ya que su función es principalmente la corrección del error.

\section{Feedback correctivo (FC)}

Como se menciona en Muñoz y Ferreira (2017), el feedback de tipo correctivo es el más comúnmente utilizado en las aulas que desarrollan el aprendizaje de una segunda lengua (L2) y uno de los más estudiados por lingüistas especializados en la materia. De acuerdo a las autoras Hernández y Reyes (2012), la relevancia del feedback correctivo en la enseñanza de una L2 puede variar según la persona docente. Tal como argumentan las autoras, esto dependería del entrenamiento en educación que cada docente haya tenido y de su propia experiencia en el área. 


\section{Feedback correctivo oral}

Ellis (2017a) y Sheen (2010a), quienes han estudiado este tipo de feedback desde hace más de una década, consideran que el feedback correctivo, ya sea oral o escrito, contribuye a la enseñanza de las lenguas, dado que las habilidades productivas son una parte fundamental. Más aún, mencionan que el feedback correctivo oral puede ser dado inmediatamente, seguido al segmento producido por el estudiantado con errores, a diferencia del feedback correctivo escrito, que difícilmente es entregado de forma inmediata. Hanh y Tho (2018) declaran que el feedback correctivo es también llamado feedback de tipo negativo, en vista de que proviene de errores lingüísticos efectuados por estudiantes en proceso de desarrollo de una lengua. Para ahondar en el tipo de feedback recién presentado, es importante mencionar que varias personas autoras han estudiado algunas estrategias del feedback correctivo oral (Sheen, 2010a, 2010b; Sheen y Ellis, 2011; Ellis 2017b).

\section{Estrategias del Feedback correctivo oral}

Numerosos estudios en este campo de la lingüística (Lyster y Ranta, 1997; Lyster y Saito, 2010; Sheen, 2010a) han examinado y planteado formas de retroalimentar estudiantes frente a errores en una L2. Lyster y Ranta (1997) distinguen cinco tipos de feedback: corrección explícita, recast, solicitud de aclaración, feedback metalingüístico y elicitación. Esta taxonomía fue evaluada por Lyster y Saito (2010), quienes definieron un meta-análisis de las estrategias, donde se evidencian los efectos positivos, duraderos y eficaces de la taxonomía para la corrección de errores.

Una de las autoras principales en esta área de la lingüística relacionada al aprendizaje de segundas lenguas es Younghee Sheen, quien ha estudiado por varios años el feedback correctivo y todas sus aristas; se ha inspirado en otros estudiosos como Lyster y Ranta (1997). Adicionalmente, en sus trabajos junto con Ellis (Sheen y Ellis, 2011), hace referencia a la taxonomia de estrategias de feedback correctivo oral .Taxonomy of Oral Corrective Feedback (CF) strategies). Esta taxonomía se basa en la entrega directa de la corrección del error (input-providing) o en un estímulo que permite que el estudiantado descubra la corrección de su error (output-prompting. En este contexto Sheen (2010a). Describe nueve tipos de FC oral, los cuales son: repetición conversacional (conversational recasts), repetición (repetition), pedir clarificación (clarification requests), repetición didáctica (didactic recasts), corrección explícita (explicit correction only), corrección explícita con explicación metalingüística (explícit correction with metalinguistic explanation), pista metalingüística (metalinguistic clue), obtención (elicitation) y signo paralingüístico (paralinguistic signal).

Dicha taxonomía se detalla en la Tabla 1 a continuación. 
TABLA 1

Taxonomía de estrategias de Feedback Correctivo Oral

\begin{tabular}{|c|c|c|}
\hline & Implícitas & Explícitas \\
\hline Entreg a de input & $\begin{array}{l}\text { Recasts conversacionales La } \\
\text { corrección consiste en la } \\
\text { reformulación del mensaje del } \\
\text { estudiantado con la intención de } \\
\text { resolver el problema que pueda } \\
\text { atentar con la comunicación. }\end{array}$ & $\begin{array}{l}\text { Recasts didácticos La corrección toma la forma de una } \\
\text { reformulación del mensaje del estudiantado, aun cuando no ha } \\
\text { surgido problema de comunicación. Corrección explícita La } \\
\text { corrección toma la forma de una señal directa que indica que un } \\
\text { error se ha cometido y la forma correcta es entregada. Corrección } \\
\text { explícita con explicación metalingüística Además de señalar que } \\
\text { un error se ha cometido y de entregar la forma correcta, también } \\
\text { hay un comentario metalingüístico. }\end{array}$ \\
\hline $\begin{array}{l}\text { Potenciar el } \\
\text { resultado correcto }\end{array}$ & $\begin{array}{l}\text { Repetición El mensaje erróneo } \\
\text { del estudiantado es repetido sin } \\
\text { destacar intencionalmente el } \\
\text { error. Solicitud de aclaración La } \\
\text { atención se enfoca en una } \\
\text { unidad problema de quien } \\
\text { habla, se indica que él o ella no } \\
\text { lo ha entendido. }\end{array}$ & $\begin{array}{l}\text { Pista Metalingüística Una oración metalingüística breve apunta a } \\
\text { obtener la forma correcta desde el estudiantado. Elicitación La } \\
\text { intención es obtener la corrección desde el estudiantado. Señal } \\
\text { paralingüística La intención es hacer una corrección al obtener la } \\
\text { respuesta de una manera no verbal. }\end{array}$ \\
\hline
\end{tabular}

Fuente: Sheen y Ellis (2011).

Nota: Traducido con base a Taxonomy of Oral CF Strategies

La taxonomía de Sheen y Ellis (2011) demuestra las distintas posibilidades que tiene tanto el alumnado como el grupo docente en la reparación del error. Dicha retroalimentación depende del tipo de error cometido por el estudiantado y del enfoque que predomine: comunicación o forma (Sheen y Ellis, 2011).

Una de las maneras de conocer el FC entregado al estudiantado, sobre cualquiera que sea el tema, forma, lugar o momento, es mediante sus percepciones. Las percepciones corresponden a un punto de partida para el personal docente, en la medida que se tenga conocimiento de los pensamientos o preferencias del estudiantado con base a sus necesidades (Atma y Widiati, 2016). Es importante conocer la percepción del alumnado, para así obtener conocimiento de qué considera que es relevante para lograr mejores aprendizajes hacia una segunda lengua. Viloria, Bertel y Daza (2015) mencionan que "la percepción ofrece los insumos sobre los cuales se forman las evidencias de acuerdo a los significados que se expresan en la sociedad y permite determinar las cualidades y elementos cualitativos de los objetos y la realidad" (p. 93). Las percepciones contienen la esencia de la información requerida, y corresponden al insumo para este estudio, en la medida que contengan las ideas y pensamientos del estudiantado frente al mejor tipo, modo, momento y agente del FC oral en el aprendizaje del inglés como lengua extranjera.

\section{Metodología}

\section{Pregunta de investigación}

¿Cuál es la percepción de estudiantes de tercer año medio de un establecimiento educacional de la provincia de Concepción hacia distintos tipos de feedback correctivo oral en errores de pronunciación?

\section{Supuestos}

El mejor momento para entregar feedback correctivo oral es inmediatamente terminada la actividad de producción. (Ellis, 2017a).

De los tipos de feedback correctivo oral, el estudiantado prefiere recasts (repetición conversacional), ya que es la que más conoce y la que más ha recibido en sus años de escolaridad (Ellis y Sheen, 2006). 


\section{Diseño de la investigación}

El presente estudio se lleva a cabo con un enfoque cualitativo de paradigma interpretativo bajo un diseño de estudio de caso.

\section{Muestra}

La muestra es por conveniencia, debido a que queda a criterio de la persona investigadora según sea una muestra de acceso disponible (Hernández, Fernández y Baptista, 2010). Esta considera 18 estudiantes, específicamente 8 hombres y 10 mujeres de enseñanza secundaria, provenientes de un establecimiento público de la provincia de Concepción, Chile, cuya edad promedio fluctúa entre los 16 y 17 años. El establecimiento educacional, además de ser estatal, cumple con ciertas características que lo hacen único en su contexto, ya que es uno de los liceos de excelencia denominados liceos bicentenarios, que apuntan a entregar educación pública y de calidad a estudiantes que quieran acceder a la educación universitaria. Según es planteado por la Pontificia Universidad Católica de Chile, a través de su Centro de Estudios de Políticas y Prácticas en Educación CEPPE, "la iniciativa de liceos de excelencia busca transformar otras tantas instituciones en 'ascensores sociales' lo largo del país para estudiantes destacados que hoy no están llegando a la educación superior" (CEPPE, 2010, p.3). "Estos establecimientos buscan desarrollar al máximo las potencialidades de niños y jóvenes altamente talentosos en las esferas de las artes, el deporte y la academia" (Eyzaguirre, 2016, p.8). Es por lo anterior que destaca de otros establecimientos municipales; por sus altos resultados académicos, el estudiantado ya está sometido a altos niveles de estrés y presión social, al querer mantener estos estándares de excelencia académica.

\section{Instrumento de recolección de datos}

Para recolectar la información, se utilizó una entrevista semiestructurada, la cual, según Plano y Creswell (2015), es una de las técnicas de recolección de datos más comunes en la investigación cualitativa. La pauta de entrevista se puede observar en la Tabla 2. 
TABLA 2

Pauta de entrevista semiestructurada

\begin{tabular}{|c|c|}
\hline \multicolumn{2}{|c|}{ Preguntas de inicio: } \\
\hline Importar lista & $\begin{array}{l}\text { ¿Con qué frecuencia los profesores y las profesoras de inglés te } \\
\text { han entregado retroalimentación en errores de pronunciación? } \\
\text { Cuéntame y Explica }\end{array}$ \\
\hline Importar lista & $\begin{array}{l}\text { En tu experiencia, ¿ha sido significativo el tipo de feedback que } \\
\text { has recibido? ¿Cómo ha sido? }\end{array}$ \\
\hline \multicolumn{2}{|c|}{ Entrevista semiestructurada: } \\
\hline Importar lista & $\begin{array}{l}\text { Con respecto a los tipos de feedback correctivo oral estudiados } \\
\text { en clases, ¿consideras más relevantes aquellos que son más } \\
\text { explícitos o implícitos en la corrección? ¿por qué? }\end{array}$ \\
\hline Importar lista & $\begin{array}{l}\text { ¿Qué tipo de feedback consideras más favorable para corregir } \\
\text { errores de pronunciación? Repetición o recast Explicación } \\
\text { explícita del error y su corrección Elicitación Metalingüistico } \\
\text { Importar lista ¿Podrias explicarme por qué? }\end{array}$ \\
\hline Importar lista & $\begin{array}{l}\text { De los tres aspectos de la pronunciación que te voy a describir } \\
\text { (Pourhosein, 2012), ¿cómo los ordenarias en orden de } \\
\text { relevancia? Linking (la vinculación) La entonación (intonation) La } \\
\text { acentuación (stress) Importar lista Te voy dar un ejemplo de } \\
\text { cada aspecto y así tú lo categorizas según el orden de prioridad } \\
\text { o relevancia al momento de recibir feedback. }\end{array}$ \\
\hline Importar lista & $\begin{array}{l}\text { ¿En qué momento prefieres que sea proporcionado el feedback? } \\
\text { Durante la tarea oral Inmediatamente finalizada la tarea oral Un } \\
\text { día después o más de un día. por qué? Importar lista }\end{array}$ \\
\hline \multicolumn{2}{|c|}{ Pregunta de cierre } \\
\hline Importar lista & $\begin{array}{l}\text { Desde tu perspectiva, ¿Cuál debería ser la metodología que } \\
\text { deberia seguir el profesor o la profesora en la corrección de } \\
\text { errores de pronunciación durante una actividad oral? }\end{array}$ \\
\hline
\end{tabular}

Fuente: Elaboración propia

Fuente: Elaboración propia

\section{Validación de los instrumentos de recolección de datos}

Un instrumento de recolección de datos es válido en la medida que responde a los objetivos de un estudio y a las características de sus participantes. (Soriano, 2014). De acuerdo con lo anterior, los instrumentos de recolección de datos del estudio en curso son validados bajo el criterio de juicio de personas expertas. Según Soriano (2014), "los expertos son personas cuya especialización, experiencia profesional, académica o investigativa relacionada al tema de investigación, les permite valorar, de contenido y de forma, cada uno de los ítems incluidos en la herramienta" (p. 25). Para efectos de este estudio, las personas expertas fueron cuatro docentes de inglés con grado de magíster o doctorado, parte de universidades locales. El profesorado hizo una revisión exhaustiva de los antecedentes que enmarca el estudio en conjunto con la pauta de entrevista. Destacaron la necesidad de introducir el tema del FCO a las personas participantes previo a la entrevista, ya que se consideran tecnicismos. Además, acotó algunas preguntas para evitar redundancia.

\section{Procedimiento de la toma de muestra}

En primer lugar, la aplicación de los instrumentos de recogida de información se genera en la medida que estén firmados y devueltos los consentimientos informados que fueron entregados por la persona investigadora.

En segundo lugar, al incluir temáticas técnicas respecto al feedback correctivo oral y procesos de retroalimentación propio de la enseñanza de las lenguas, es que el estudiantado fue sometido a una inducción 
básica previo a la entrevista, en donde se le dio a conocer modelos de estrategias de feedback correctivo oral (Sheen y Ellis, 2011; Hernández y Reyes, 2012; Ellis, 2017b), con el fin de aclarar dudas que puedan surgir en torno a los tecnicismos inusuales para estudiantes de entre 16 y 17 años.

Luego, se procedió a aplicar la entrevista, las cuales fueron grabadas en formato de audio.

Posteriormente, se realizó su transcripción y posterior análisis.

\section{AnÁLisis CUALITATIVo}

Las respuestas del grupo de participantes fueron analizadas mediante la técnica de análisis de contenido cualitativo, que según Kvale (2007) se relaciona con el análisis con foco en el significado. Además, involucra procesos como la codificación y la categorización para darle significado al discurso del sujeto.

\section{Etapas del análisis}

\section{Etapa 1: Primera instancia de codificación}

Una vez efectuada la transcripción de las 18 entrevistas, se procedió a la codificación entrevista por entrevista según las categorías apriorísticas establecidas desde la literatura y que ayudaron a la conformación de la pauta de entrevista. Como expresa Cisterna (2005), "las categorías apriorísticas son construidas antes del proceso recopilatorio de información o emergentes, que surgen desde el levantamiento de referenciales significativos a partir de la propia indagación”. (p. 64).

La Tabla 3 muestra cómo se efectuó la codificación entrevista por entrevista a partir de las categorías apriorísticas. 
TABLA 3

Extracto de codificación en primera instancia

\begin{tabular}{|c|c|}
\hline & Categorias apriorísticas \\
\hline & $\begin{array}{l}\text { Frecuencia en la entrega de retroalimentación en errores de } \\
\text { pronunciación. }\end{array}$ \\
\hline Entrevista 13 & $\begin{array}{l}\text { Sí, me han hecho retroalimentación, pero muy pocas veces (R-nf) de } \\
\text { pronunciación, siempre o casi siempre lo anotan en la prueba o no lo } \\
\text { hacen, así que tengo que buscar la respuesta yo mismo. (R-sns) }\end{array}$ \\
\hline Entrevista 14 & $\begin{array}{l}\text { Eh, en el momento de las clases, eh, siempre que teníamos actividades } \\
\text { donde teniamos que hablar, como, si lo tuviera que medir como en } \\
\text { tiempo, serian como dos veces al mes, en la cual me pedía o leer o } \\
\text { cualquier actividad y mientras, si yo pronunciaba una palabra, me la } \\
\text { corregía en el momento. (R-f) (R-eo) }\end{array}$ \\
\hline Entrevista 15 & $\begin{array}{l}\text { Eh, no me han corregido mucho lo que llevo en el bicentenario, parece } \\
\text { que mi pronunciación es bastante buena. (R-nf) }\end{array}$ \\
\hline Entrevista 16 & $\begin{array}{l}\text { En las presentaciones orales, más que nada, al momento de hablar, } \\
\text { luego de terminar las presentaciones orales, ( } \mathrm{R}-\mathrm{e} \text { ) eh, se, me remarcan } \\
\text { como los errores que tuve en pronunciaciones (C-ve) y cosas asi. Más } \\
\text { allá no, dependiendo, a veces, de las preguntas que me hacen, eh, en } \\
\text { clases o cosas asi. }\end{array}$ \\
\hline Entrevista 17 & $\begin{array}{l}\text { A ver, cuando tenemos alguna presentación oral, cuando estamos, } \\
\text { hacemos los speaking, por lo general, en esos momentos me } \\
\text { retroalimentan (R-eo) cuando me equivoco en alguna palabra o } \\
\text { cuando le preguntamos directamente, eh, sobre eso. O cuando estamos } \\
\text { en alguna prueba, también, hay veces que le preguntamos la } \\
\text { pronunciación de una palabra para poder entender mejor el contexto. } \\
\text { (R-f) }\end{array}$ \\
\hline Entrevista 18 & $\begin{array}{l}\text { (...) normalmente cuando hay speaking o presentaciones tengo que } \\
\text { corregir una o dos palabras(R-eo) solamente, pero no es muy seguido } \\
\text { (R-nf). }\end{array}$ \\
\hline
\end{tabular}

Fuente: Elaboración propia

\section{Asignación de códigos, construcción del libro de códigos}

La asignación de códigos nace del significado de la respuesta de cada estudiante. De acuerdo con Miles, Huberman y Saldaña (2014), la codificación significa realizar un análisis que requiere de inferencias y reflexiones profundas.

A medida que estos códigos se van asignando, se van registrando en un documento aparte llamado libro de códigos. Este libro de códigos contiene todos los códigos recolectados en esta primera instancia de análisis, la descripción de cada uno y la frecuencia (número de veces que se hace alusión a la temática, punto de vista y opinión que el código representa).

A continuación, en la Tabla 4, se ilustra un ejemplo del libro de códigos inicial

TABLA 4

Extracto del libro de códigos inicial

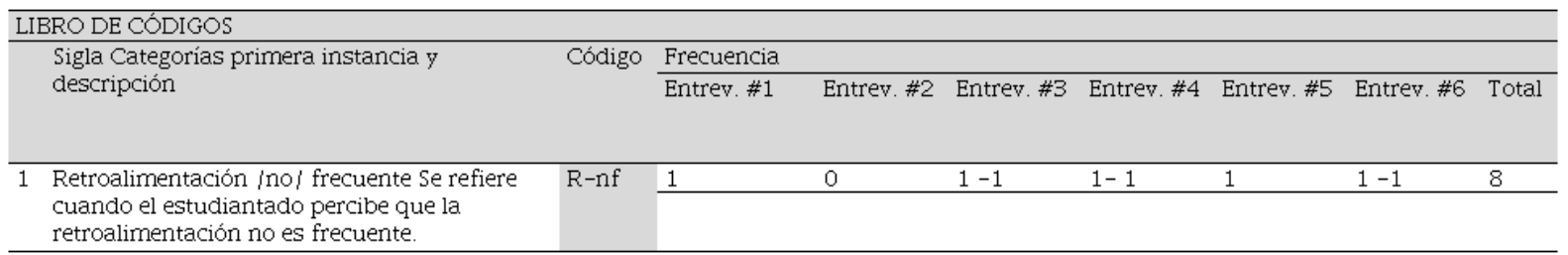




\section{Etapa 2: Revisión de códigos y construcción de codificación de segundo orden}

En esta etapa se renombran, fusionan o eliminan códigos hasta que exista una mejor representación del patrón de significado que está en construcción. Según Gibbs (2012), la codificación de segundo orden corresponde al cambio de nivel en la codificación, en donde el análisis se centra en la formulación futura de categorías o se piensa en afianzar las categorías establecidas a modo preliminar.

A continuación, en la Tabla 5, se presenta un extracto de la matriz de codificación de segundo orden.

TABLA 5

Extracto de matriz de codificación de segundo orden

\begin{tabular}{|c|c|c|c|}
\hline & Código & Código inicial & Frec \\
\hline 1 & $\mathrm{R}-\mathrm{nf}$ & Retroalimentación /no/ frecuente & 11 \\
\hline 2 & $R-f$ & Retroalimentación/frecuente & 5 \\
\hline 3 & R-eo & Retroalimentación/evaluaciones/orales & 11 \\
\hline 4 & R-sns & $\begin{array}{l}\text { Retroalimentación/superficial /no/ } \\
\text { suficiente }\end{array}$ & 4 \\
\hline 5 & R-e & Retroalimentación/efectiva & 18 \\
\hline & $R-n b$ & Retroalimentación /no/ beneficiosa & 1 \\
\hline
\end{tabular}

\begin{tabular}{|c|c|c|c|}
\hline & Código & Códigos inicial & Frec \\
\hline 3 & R-eo & Retroalimentación/evaluaciones/orales & 11 \\
\hline & R-sns & $\begin{array}{l}\text { Retroalimentación/superficial /no/ } \\
\text { Suficiente }\end{array}$ & 16 \\
\hline & R-e & Retroalimentación/efectiva & 23 \\
\hline
\end{tabular}

Nota: Se fusionaron los códigos 1, 4 y 6 con el código 4

Retroalimentación/superficial/no/suficiente se

fusionaron los códigos 2 y 5 con el código 5

Retroalimentación/efectiva

Fuente: Elaboración propia

De este modo se redujo de 31 a 14 códigos, los cuales conforman el libro de códigos de segundo orden. Además, se realiza la identificación de categorías que, a su vez, subyacen de la teoría y corresponde a la etapa que se describe a continuación.

\section{Etapa 3: Confección de matriz de categorias y subcategorias}

Los códigos finales dan significado a las categorías y sus subcategorías, las cuales están también vinculadas con la teoría que enmarca este estudio.

A continuación, en la Tabla 6 , se presenta un extracto de la matriz de categorías y subcategorías.

TABLA 6.

Extracto de matriz de categorías y subcategorías

\begin{tabular}{ll}
\hline lera categoría: rol del feedback \\
\hline Subcategorias & $\begin{array}{l}\text { Lo hacemos sólo como cuando tenemos como evaluaciones orales tipo presentación, interacciones } \\
\text { en parejas de situación o cuando nos toca exponer una disertación sobre algún tema, ahi es } \\
\text { donde vemos un poco más la parte oral } \mathrm{E} 1 \text { La mayoría de las veces es cuando se realiza una } \\
\text { evaluación oralo luego de eso } \mathrm{E} 2\end{array}$ \\
\hline $\begin{array}{l}\text { Feedback en evaluaciones } \\
\text { orales }\end{array}$ \\
\hline
\end{tabular}




\section{Proceso de triangulación}

En este estudio se implementó una triangulación de fuentes, donde se consideró a cada participante como fuente distinta de información (Ruiz-Olabuénaga, 2003). Además, se llevó a cabo la comprobación de los resultados mediante el software NVivo 12 a los días después de lo realizado por la persona investigadora.

\section{Resultados}

La Tabla 7 muestra las categorías y subcategorías que emergieron a partir del análisis de la información obtenida en las entrevistas al estudiantado.

TABLA 7

Categorías y subcategorías

\begin{tabular}{|c|c|c|}
\hline \multicolumn{2}{|c|}{ Categorías } & \multirow{2}{*}{$\begin{array}{l}\text { Subcategorías } \\
\text { Feedback en evaluaciones } \\
\text { orales }\end{array}$} \\
\hline 1 & Rol del feedback & \\
\hline & & Feedback no suficiente \\
\hline & & Feedback efectivo \\
\hline \multirow[t]{2}{*}{2} & \multirow{2}{*}{$\begin{array}{l}\text { Consecuencias del } \\
\text { feedback }\end{array}$} & Visibilizar errores \\
\hline & & Beneficiar la pronunciación \\
\hline \multirow[t]{2}{*}{3} & \multirow{2}{*}{$\begin{array}{l}\text { Preferencia por modo de } \\
\text { feedback }\end{array}$} & Feedback implícito \\
\hline & & Feedback explícito \\
\hline \multirow[t]{3}{*}{4} & \multirow{3}{*}{$\begin{array}{l}\text { Preferencia por estrategia } \\
\text { de feedback }\end{array}$} & Estrategia metalingüística \\
\hline & & $\begin{array}{l}\text { Estrategia explicación } \\
\text { explícita }\end{array}$ \\
\hline & & Estrategia repetición o recast. \\
\hline \multirow[t]{2}{*}{5} & \multirow{2}{*}{$\begin{array}{l}\text { Importancia de la } \\
\text { pronunciación }\end{array}$} & Feedback de la acentuación \\
\hline & & Pronunciación relevante \\
\hline \multirow[t]{2}{*}{6} & Momento de feedback & Feedback inmediato \\
\hline & & Feedback que interrumpe \\
\hline
\end{tabular}

Fuente: Elaboración propia

\section{Distribución general de las categorías}

A modo global, las categorías quedan distribuidas de la siguiente manera, según sus porcentajes de cobertura en la muestra arrojados por el software NVivo 12. 


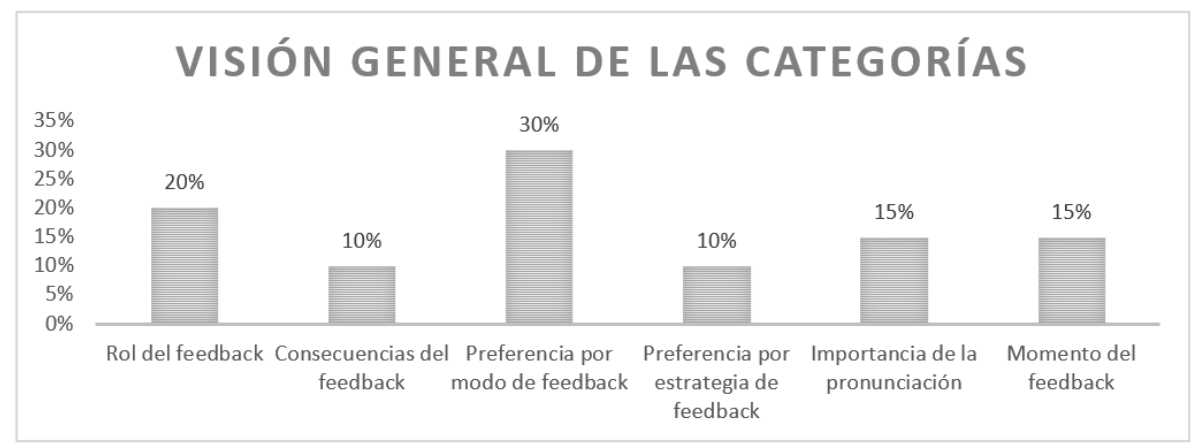

FIGURA 1.

Visión general de las categorías

Fuente: Elaboración propia

La Figura 1 ilustra que la mayor opinión se encontró en relación a la categoría 3, preferencia por modo de feedback, y las menos mencionadas fueron las categorías 2 y 4, consecuencias del feedback y preferencia por estrategia de feedback. Es decir, el estudiantado habló de forma concisa respecto a sus opiniones, las cuales fueron variadas.

A continuación, se presentan los hallazgos más relevantes en función de las unidades de significado mencionadas anteriormente.

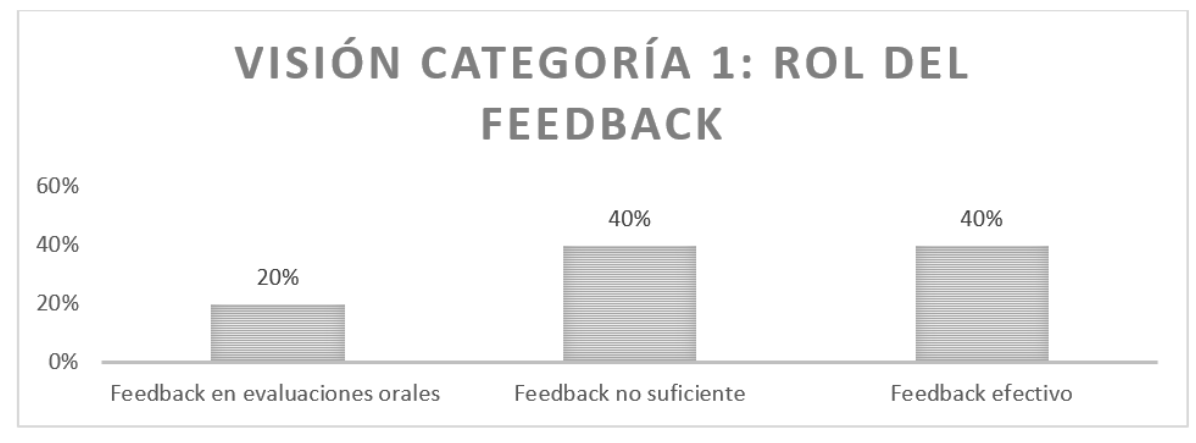

FIGURA 2.

Visión categoría 1: Rol del feedback

Fuente: Elaboración propia

En general, se identifica que la retroalimentación es un concepto medianamente conocido por el estudiantado, pero que solo aparece en el aspecto académico cuando realizan alguna evaluación oral determinada. La Figura 2 demuestra que el alumnado se refiere bastante a que el feedback recibido a la fecha no ha sido suficiente.

\section{Categoría 2: Consecuencias del feedback}

Las subcategorías de esta categoría se presentan de forma pareja en la muestra. Las personas participantes declaran que el feedback les beneficiará tanto para la pronunciación como para la visualización de sus errores, aspectos que benefician el desarrollo de la producción del idioma. Esto se visualiza en la Figura 3. 


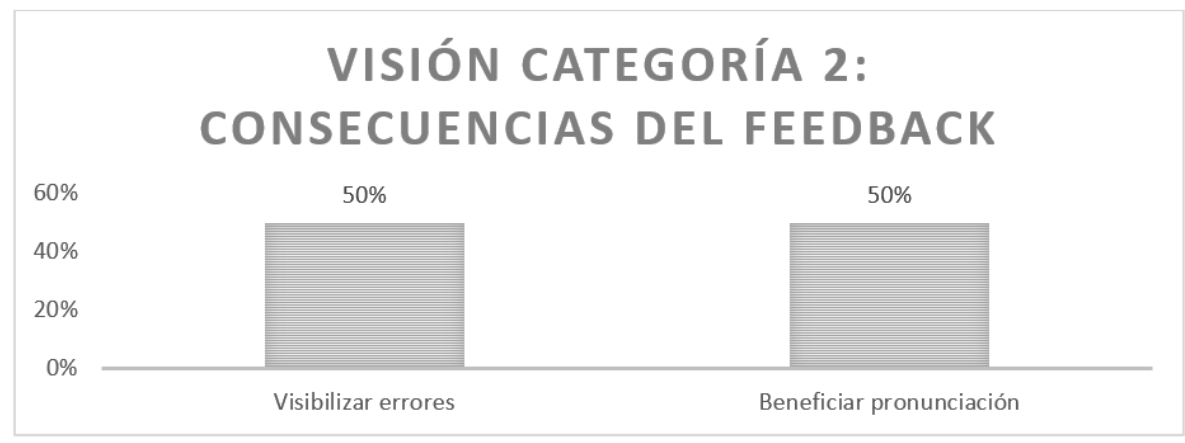

FIGURA 3.

Visión categoría 2: Consecuencias del feedback

Fuente: Elaboración propia

\section{Categoría 3: Preferencia por modo de feedback}

La retroalimentación según modo considera la retroalimentación de tipo explícita, donde la corrección es entregada directamente al estudiantado, y la de tipo implícita, donde la respuesta o corrección de los errores es insinuada al estudiantado, de modo que este pueda procesarlo y autocorregirse. Cabe mencionar que este último modo de retroalimentación es el predominante conforme a las frecuencias y es la primera subcategoría a ser definida dentro de esta categoría. Esto se visualiza en la Figura 4.

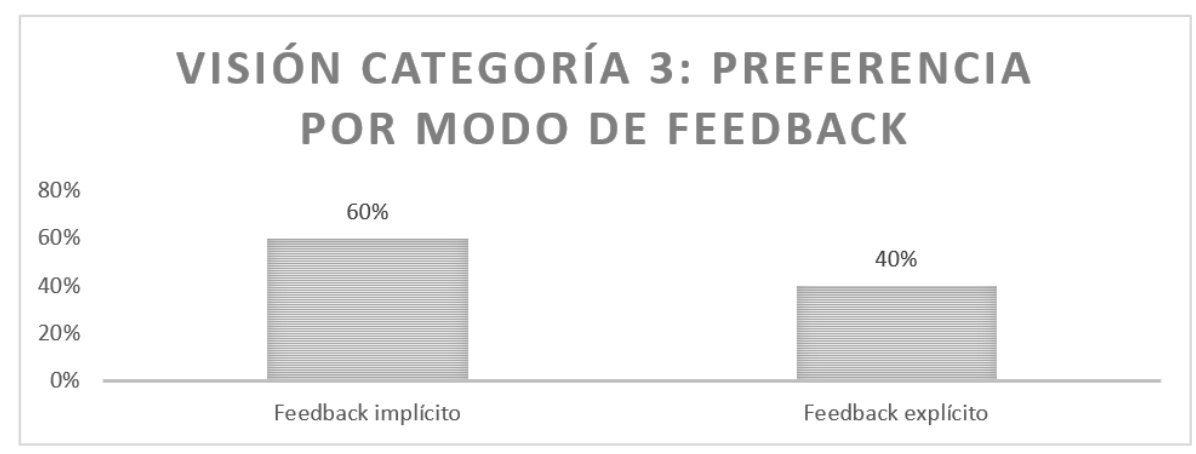

FIGURA 4.

Visión categoría 3: Preferencia por modo de feedback

Fuente: Elaboración propia

\section{Categoría 4: Preferencia por estrategia de Feedback Correctivo Oral (FCO). (Sheen y Ellis, 2011)}

Al estudiantado del estudio se le presentó la posibilidad de elegir algunas estrategias de Feedback Correctivo Oral (FCO) aportadas desde la literatura de los autores Sheen y Ellis (2011). De las estrategias presentadas, las más preferidas por el grupo estudiantes fueron tres: estrategia de explicación explícita del error y su corrección, la estrategia pista metalingüística y la estrategia de repetición, también llamada recast. Se visualiza en la Figura 5 que la más elegida es la de tipo pista metalingüística, la cual, aunque esté clasificada como de tipo explícito según la taxonomía de Sheen y Ellis (2011), considera una forma implícita de corrección, ya que no entrega la respuesta explícitamente. 


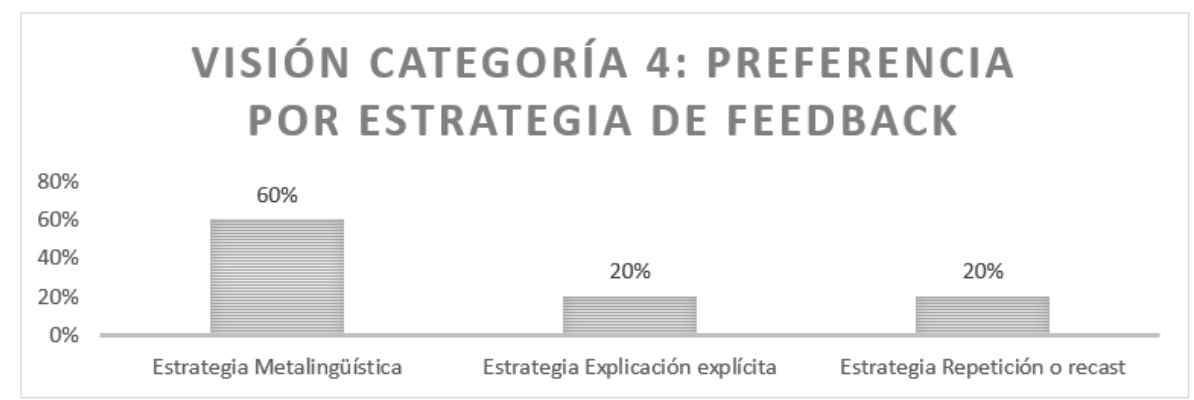

FIGURA 5.

Visión categoría 4: Preferencia por estrategia de feedback

Fuente: Elaboración propia

\section{Categoría 5: Importancia de la pronunciación}

La pronunciación es un aspecto importante de aprender y, por ende, de ser retroalimentado en las clases de inglés. En consecuencia, es considerada primordial a la hora de aprender un segundo idioma, dado que permite entregar mensajes claros y que promueven la comunicación efectiva. Dentro de esta categoría se encuentra la subcategoría de preferencia de uno de los sub-aspectos de la pronunciación, como lo es la acentuación (Pourhosein, 2012) y el rol relevante de la pronunciación en la ejecución de un mensaje oral. Esto último es lo más valorado de esta categoría por el estudiantado, según se ilustra en la Figura 6.

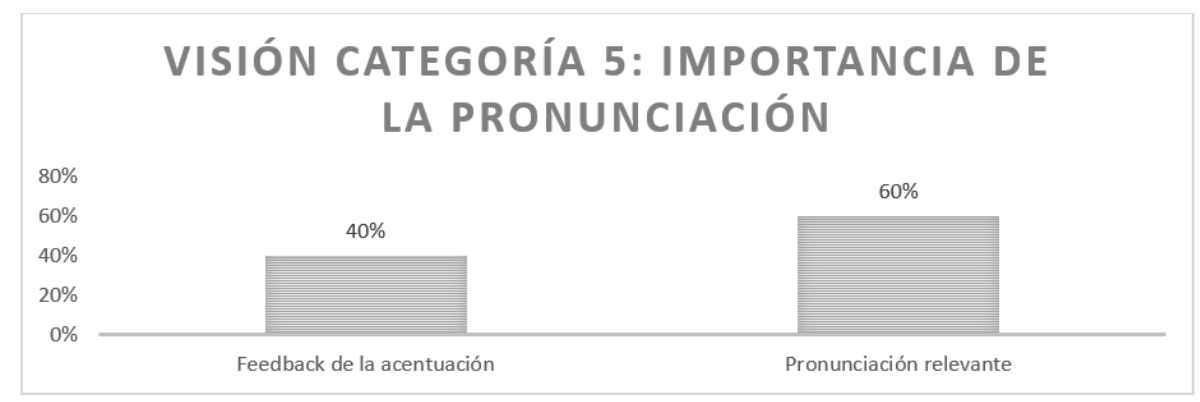

FIGURA 6.

Visión categoría 5: Importancia de la pronunciación

Fuente: Elaboración propia

\section{Categoría 6: Momento de entrega del FCO}

En relación al momento en que el estudiantado prefiere recibir el feedback, se presentaron las siguientes opciones: a) inmediatamente terminada una tarea oral, b) durante la tarea oral, c) al tiempo después. Para ello, el momento preferido de entrega de feedback es inmediatamente terminada la tarea oral, puesto que durante la ella se le interrumpe y no puede efectuar el proceso de aprendizaje correctamente. Esto se visualiza en la Figura 7. 


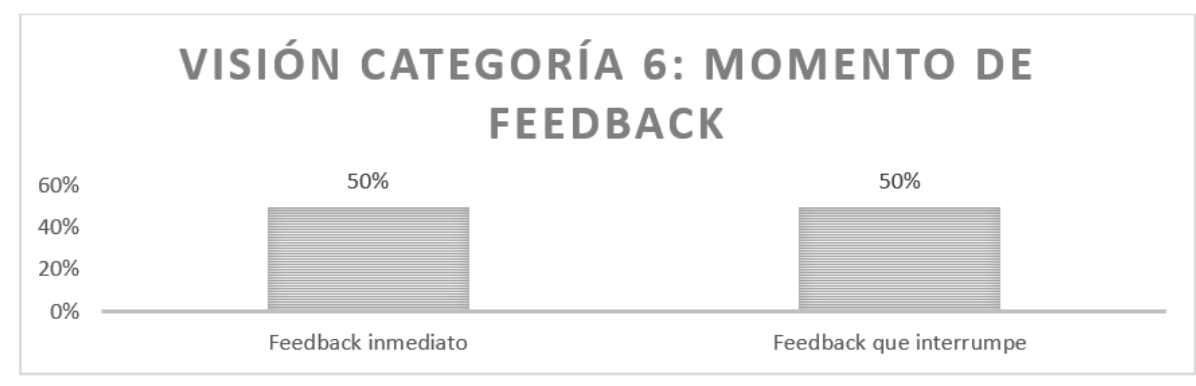

FIGURA 7.

Visión categoría 6: Momento de feedback

Fuente: Elaboración propia

\section{Discusión}

Se evidencia una percepción general positiva frente al feedback. El estudiantado considera que, aunque las instancias de retroalimentación son puntuales y escasas, estas de igual manera han sido efectivas para mejorar errores y así avanzar en sus aprendizajes. Según Shabani y Safari (2016), el conocer la forma de retroalimentar ayuda a que el estudiantado tenga una buena actitud hacia el idioma. Para que la retroalimentación resulte beneficiosa, se deben manejar ciertas estrategias para poder transmitir esa información y que pueda ser usada por el alumnado, tal como lo mencionan Contreras y Zúñiga (2017). Para las personas participantes, la retroalimentación o tipos de feedback correctivo oral logran visibilizar sus errores y también beneficiar aspectos puntuales de la oralidad, como en el caso de la pronunciación.

Tras el análisis efectuado, se demuestra que el estudiantado no desea que se le entreguen las respuestas; ya que el hecho de procesar cuál fue su error hace que el aprendizaje sea más significativo, porque existe un proceso interno que convierte al estudiantado en el actor principal. Esto hace sentido cuando se pretende considerar al alumnado como centro de su propio aprendizaje (MINEDUC, 2019a).

Si bien los autores Hernández y Reyes (2012) mencionan que es decisión del profesorado la elección del modo de feedback a entregar, es interesante saber que la opinión del estudiantado es aún más valiosa. Tal como lo mencionan Huang y Jia (2016), quienes consideran que, si bien es favorable que tanto docentes como estudiantes decidan acerca del modo de entrega del feedback, esto no significa que el estudiantado no cometa los mismos errores nuevamente, sino que es todo parte del proceso de aprendizaje. Es por esto que el foco está puesto en cómo el estudiantado considera que aprenderá mejor.

Las personas participantes determinaron que el de tipo pista metalingüística es la más favorable para sus aprendizajes, debido a que la entrega de la respuesta no es directa, sino que esta cuenta con información clave que le hace pensar de manera eficaz en mejorar sus respuestas. Ellis (2017a) menciona que la estrategia metalingüística de $F C O$ provee al estudiantado de comentarios que pueden ser extendidos, que emergen de una suerte de tratamiento que va más allá de la mera corrección. Al ser este el feedback más preferido por el estudiantado, se contradice a los estudios de Ellis y Sheen (2006), ya que mencionan que la estrategia comúnmente preferida por estudiantes es recast. Se infiere entonces que si el alumnado fuera consciente de todas las estrategias de feedback correctivo oral que entrega la teoría (Sheen y Ellis, 2011), este podría optar por diversas formas de ser retroalimentado según a sus estilos de aprender, su ritmo de aprendizaje, su nivel de inglés, entre otros factores.

Al hablar de corregir errores de pronunciación mediante el FCO, los sujetos consideraron que el feedback enfocado hacia errores de acentuación contribuye a la mejora de la pronunciación. Huang y Jia (2016) afianzan esto al considerar que el feedback correctivo puede ayudar enormemente a la acentuación de palabras. El autor Pourhosein (2012) menciona que es importante desarrollar la pronunciación del estudiantado, ya que "una pronunciación limitada puede disminuir la confianza en sí mismos, restringir las interacciones 
sociales y afectar negativamente sus habilidades" (p. 119). Esto evidentemente afecta aspectos motivacionales, incluso cuando se quiere que el alumnado tenga una actitud positiva para enfrentar el aprendizaje de una lengua extranjera. Dicho esto, si se potencia el mejoramiento de la pronunciación, se contribuye a la formación de personas seguras de sí mismas y capaces de enfrentar contextos de comunicación real en un segundo idioma.

Desde el análisis también queda en evidencia que el mejor momento, según el estudiantado, para recibir feedback correctivo oral, cualquiera sea el tipo, es inmediatamente terminada la tarea oral. De igual forma, Huang y Jia (2016) determinan que la entrega de feedback durante la tarea interrumpe su proceso de producción, lo cual puede afectar tanto al mensaje que se está emitiendo como al feedback que se les está entregando. Shabani y Safari (2016) mencionan que el cuándo entregar feedback correctivo oral "afecta los niveles de ansiedad de los estudiantes mientras ellos están participando de actividades orales” (p. 2224). También establecen que la persona docente debe decidir el momento de entrega dependiendo de las necesidades y características particulares de su grupo de estudiantes. Esto levanta interés, puesto que le da sentido al por qué es necesario conocer las percepciones del alumnado en relación con los aspectos del feedback correctivo oral, como lo es el momento. Cabe decir que es importante considerar que la decisión del momento puede ser consensuada previo a la tarea oral con el estudiantado, esto en consideración con la variación de necesidades, objetivos, gustos y preferencias respecto a esto.

\section{Limitaciones}

Una de las primeras limitaciones para realizar este estudio fue el estallido social ocurrido en Chile en octubre de 2019, ya que para esa fecha estaba planificado efectuar la toma de muestra. Esto no se pudo llevar a cabo ya que las clases fueron suspendidas a nivel nacional como medida de seguridad para el estudiantado.

Otro de los factores que pudo haber interferido en el correcto desarrollo de este estudio fue la pandemia vivida a nivel mundial desde marzo de 2020, ya que era el momento de reiniciar con la toma de muestra. Esto tuvo que ser realizado vía online, lo que permitió concretar las entrevistas.

Un tercer factor que interfirió en el ideal desarrollo de este estudio es la edad de las personas participantes, ya que como son jóvenes de entre 16 y 17 años de edad, su capacidad de análisis y de profundizar ciertos temas fue bastante limitada, además que sus opiniones podrían haber estado afectadas por factores anexos, como timidez, falta de sueño o influencias de sus compañeras y compañeros en querer responder algo similar. Aunque la entrevista se hubiera realizado de manera individual, el estudiantado de igual forma comenta o habla acerca de lo que respondió cada persona.

Por último, un cuarto factor que pudo haber limitado este estudio es la falta de estudios nacionales en relación a esta temática, ya que lo que existe está particularmente dirigido en conocer las percepciones del profesorado en torno al feedback correctivo.

\section{Proyecciones}

Las proyecciones de estudio sugieren lo siguiente:

- Investigar acerca del efecto o impacto del feedback correctivo oral en errores de pronunciación mediante un estudio experimental con grupos de control con implicancias cuantitativas para abordar una mayor cantidad de muestra.

- Realizar un estudio comparativo entre el actual estudio de enfoque cualitativo y el propuesto de orden cuantitativo para llegar a conclusiones reforzadas. 
- Investigar acerca de las percepciones del profesorado de inglés, de establecimientos educacionales de orden público, en relación a las estrategias del feedback correctivo oral en errores de pronunciación.

- Aumentar el alcance de la investigación hacia distintos tipos de errores, como gramaticales o según distintos niveles de inglés del estudiantado.

\section{Conclusiones}

A lo largo de este estudio se evidenció que el feedback es un aspecto del proceso educativo que el estudiantado conoce a modo general, más no específico, ya que desconoce particularidades del concepto, por ejemplo, el feedback correctivo oral. Al determinar la preferencia por el modo implícito de corrección, el estudiantado alude a que ello le permite pensar la respuesta, activar aprendizajes previos y ejercer la autocorrección, lo que evidentemente le beneficia a su aprendizaje y permite que lo puedan recordar a futuro. Ante los tipos de feedback correctivo oral presentados, el estudiantado prefirió el de tipo metalingüístico, de lo que se concluye que una parte del grupo de estudiantes prefiere conocer la naturaleza del error.

En este estudio, la pronunciación es considerada como algo relevante de potenciar, particularmente el subaspecto de la acentuación. En este contexto, aprender inglés bajo el enfoque comunicativo, que promueve el Ministerio de Educación, permite la expresión de mensajes comprensibles, no precisamente perfectos. Para finalizar, la pronunciación es un aspecto complejo y de mejora constante, por lo tanto, los errores a cometer por el estudiantado pueden seguir ocurriendo en el futuro, es por ello la importancia de la provisión de feedback.

\section{REFERENCIAS BIBLIOGRÁFICAS}

Aránguiz, M. y Quintanilla, A. Q. (2016). Oral corrective feedback strategies in EFL. A pilot study in Chilean classrooms/estrategias correctivas orales en inglés como lengua extranjera. Un estudio piloto en las aulas chilenas. ELIA: Estudios de Lingüistica Inglesa Aplicada, 16, 103-132. Recuperado de http://revistas.uned.es/index.php /ELIA/article/view/17947

Atma, N. y Widiati, U. (2016). EFL students' preferences for corrective feedback in speaking instruction across speaking course levels. Bahasa dan Seni, 43(2), 183-195. Recuperado de http://journal2.um.ac.id/index.php/j bs/article/view/194

Baker, A.A. y Burri, M. (2016). Feedback on second language pronunciation: A case study of EAP teachers' beliefs and practices. Australian Journal of Teacher Education, 41, 1-19. Recuperado de https://eric.ed.gov/?id=EJ1112389

Bohn, H. y Hansen, T. (2017). Assessing pronunciation in an EFL context: Teachers' orientations towards nativeness and intelligibility. Language Assessment Quarterly, 14(1), 54-68. doi: https://www.tandfonline.com/doi/abs/1 $0.1080 / 15434303.2016 .1256407$

Brown, H.D. (2001). Teaching by Principles: an Interactive Approach to Language Pedagogy. White Plains, NY: Longman. doi: https://doi.org/10.2307/3587655

Canabal, C. y Margalef, L. (2017). La retroalimentación: la clave para la evaluación orientada al aprendizaje. Profesorado, 2, 149-170 Recuperado de https://digibug.ugr.es/handle/10481/47669

CEPPE (2010). Liceos de Excelencia: Ideas y experiencias relevantes para su diseño e implementación, Notas de Educación. 3, 1-10. Recuperado de http://200.6.99.248/ bru487cl/files/Liceos_Excelencia\%5B1\%5D.pdf

Cisterna, F. (2005). Categorización y triangulación como procesos de validación del conocimiento en investigación cualitativa. Theoria, 14(1), 61-71. Recuperado de https://www.redalyc.org/pdf/299/29900107.pdf

Contreras, G. y Zúñiga, C. (2017). Concepciones de profesores sobre retroalimentación: Una revisión de la literatura. Magis, Revista Internacional de Investigación en Educación, 9(19), 69-90. Recuperado de https://www.redalyc. org/pdf/2810/281052678004.pdf 
Ellis, R. y Sheen, Y. (2006). Re-examining the role of recasts in second language acquisition. Studies in Second Language Acquisition, 28(4), 575-600. Recuperado de https://eric.ed.gov/?id=EJ777403

Ellis, R. (2017a). Oral corrective feedback in L2 classrooms: What we know so far. En H. Nassaji y E. Kartchava (Eds.), Corrective feedback in second language teaching and learning: Research, theory, applications, implications, (pp.19-36). New York, NY: Routledge. doi: https://doi.org/10.4324/9781315621432

Ellis, R. (2017b). Oral corrective feedback in language teaching: A historical perspective. Avances En Educación $Y$ Humanidades, 2(2), 7-22. doi: https://doi.org/10.21897/25394185.1482

Eyzaguirre, S. (2016). ¿Por qué Liceos de Excelencia? Revista Puntos de Referencia. Centro de Estudios públicos. 448, 1-18. Recuperado de https://bit.ly/3t7zlmE

Gibbs, G. (2012). El análisis de datos cualitativos en investigación cualitativa: Graham Gibbs. Madrid: Morata. Recuperado de https://bit.ly/2GlwmUV

Hanh, P. y Tho, P. (2018). Oral corrective feedback in EFL/ESL classrooms: Classification models. VNU Journal Of Foreign Studies, 34(5), 40-48. Recuperado de https://js.vnu.edu.vn/FS/article/view/4301

Hattie, J. y Timperley, H. (2007). The power of feedback. Review of educational research, 77(1), 81-112. Recuperado de https://bit.ly/3n6JGxo

Hernández, R., Fernández. C y Baptista, P. (2010). Metodología de la Investigación, (Quinta Edición). México: McGraw-Hill Interamericana. Recuperado de http://www.ru.tic.unam.mx:8080/handle/123456789/1825

Hernández, E. y Reyes, M. (2012). Teachers' perceptions about oral corrective feedback and their practice in EFL classrooms. Profile Issues in Teachers Professional Development, 14(2), 63-75. Recuperado de https://bit.ly/2G $9 \mathrm{FVq} 8$

Huang, X. y Jia, X. (2016). Corrective Feedback on Pronunciation: Students' and Teachers' Perceptions. International Journal of English Linguistics, 6(6), 245-254. doi: https://doi.org/10.5539/ijel.v6n6p245

Kenworthy, J. (1987). Teaching English Pronunciation. Londres y Nueva York: Longman. Recuperado de https://b it.ly/3vjAhpo

Kvale, S. (2007). Doing interviews. London: Sage. doi: https://dx.doi.org/10.4135/9781849208963

Lyster, R., y Ranta, L. (1997). Corrective feedback and learner uptake. Studies in Second Language Acquisition, 19, 37 66. Recuperado de https://bit.ly/3eGlrTe

Lyster, R. y Saito, K. (2010). Oral feedback in classroom SLA: A meta-analysis. Studies in second language acquisition, 32, 265-302. Recuperado de https://bit.ly/3vsPnsy

Marzá, N. (2014). Pronunciation and comprehension of oral English in the English as a foreign language class: Key aspects, students' perceptions and proposals. Journal of Language Teaching and Research, 5(2), 262-273. doi: h ttps://doi.org/10.4304/jltr.5.2.262-273

Ministerio de educación chileno. (2019a). Nuevo Curriculum $3^{\circ}$ y $4^{\circ}$ medio. Unidad de Currículum y Evaluación UCE. Santiago: MINEDUC. Recuperado de https://bit.ly/2GiO8If

Ministerio de educación chileno. (2019b). Bases Curriculares $3^{\circ}$ y $4^{\circ}$ medio. Santiago: MINEDUC. Recuperado de h ttps://bit.ly/2EIIVcf

Miles, M. B., Huberman, A. M. y Saldaña, J. (2014). Qualitative data analysis: A methods sourcebook (Third edition.). Thousand Oaks, California: SAGE Publications, Inc. Recuperado de https://eric.ed.gov/?id=ED565763

Muñoz, B. y Ferreira, A. (2017). El Feedback Correctivo Escrito Indirecto en el Aprendizaje de la Forma Comparativa de Adjetivos en Inglés. Logos: Revista de Lingüistica, Filosofía y Literatura, Barcelona, 27(1), 73-89. Recuperado de https://bit.ly/3jg7yw2

Nunan, D. (2003). Practical English language teaching. New York: McGraw-Hill/Contemporary. Recuperado de ht tps://bit.ly/2Gmvi2O

Plano, V.L. y Creswell, J.W. (2015). Understanding Research: A consumer's guide. $2^{\text {nd }}$ edition. London, UK: Pearson. Recuperado de https://bit.ly/33fE5N8

Poulos, A. y Mahony, M.J. (2008). Effectiveness of feedback: The students' perspective. Assessment and Evaluation in Higher Education, 33(2), 143-154. doi: https://doi.org/10.1080/02602930601127869 
Pourhosein, A.G. (2012). A study of factors affecting EFL learners\# English pronunciation Learning and the strategies for instruction. The International journal of Humanities and Social Sciences, 2(3), 119-128). Recuperado de htt p://ijhssnet.com/journals/Vol_2_No_3_February_2012/17.pdf

Quinn, P. y Nakata, T. (2017). The timing of oral corrective feedback. En H. Nassaji y E. Kartchava (Eds.), Corrective feedback in second language teaching and learning: Research, theory, applications, implications (pp. 35-47). Abingdon, UK: Routledge. Recuperado de https://scholar.google.com/scholar?oi=bibs\&hl=id\&cluste $\mathrm{r}=12836719597277449348$

Ruiz - Olabuenaga, J. (2003). Técnicas de triangulación y control de calidad en la investigación socioeducativa. Bilbao: Universidad de Deusto. Recuperado de https://dialnet.unirioja.es/servlet/libro?codigo=326747

Selinker, L. (1972). Interlanguage. International Review of Applied Linguistics, 10, 209-31.

Shabani, K. y Safari, F. (2016). Immediate vs Delayed Correction Feedback (CF) and Accuracy of Oral Production: The Role of Anxiety. Theory and Practice in Language Studies, 6, 2222-2230. doi: https://dx.doi.org/10.17507 /tpls.0611.21

Sheen, Y. (2010a). The role of oral and written corrective feedback in SLA. Studies in Second Language Acquisition, 32(2), 169-179. doi: https://doi.org/10.1017/S0272263109990489

Sheen, Y. (2010b). Differential effects of oral and written corrective feedback in the ESL classroom. Studies in Second Language Acquisition, 32(2), 201-234. doi: https://doi.org/10.1017/S0272263109990507

Sheen, Y. y Ellis, R. (2011). Corrective feedback in language teaching. En E. Hinkle (Ed.), Handbook of research in second language teaching and learning (2), 593-610. New York, NY: Routledge. Recuperado de https://espace. curtin.edu.au/handle/20.500.11937/60482

Soriano, A. M. (2014). Diseño y validación de instrumentos de medición. Diálogos. 14, 19-40. Recuperado de http:/ /www.lamjol.info/index.php/DIALOGOS/article/view/2202/1997

Ur, P. (2009). A course in English language teaching. Cambridge, England: Cambridge University Press. Recuperado de https://bit.ly/2SemcYA

Viloria, J., Bertel, M. y Daza, A. (2015). Percepciones estudiantiles sobre el proceso de acreditación por alta calidad del programa de administración de empresas de la Universidad del Magdalena. Praxis, 11, 89-102. doi: https:/ /doi.org/10.21676/23897856.1557

\section{INFORMACIÓN ADICIONAL}

Cómo citar: Orellana Marihuén, P.F. y Ortiz Navarrete, M.A. (2021). Percepción del estudiantado chileno de enseñanza secundaria hacia distintos tipos de Feedback Correctivo Oral (FCO) en errores de pronunciación: un análisis cualitativo. Revista Educación, 45(2). Recuperado de http://doi.org/10.15517/revedu.v45i1.43 636 